\title{
Full intuitive creativity is a collective work of the quantum computer of our subconscious and the digital computer of our conscious
}

\begin{abstract}
We are living in the $21^{\text {st }}$ century and we still don't understand how intuitive creativity works-our science cannot explain it. At the same time, this is the type of creativity that moves the development of our civilization ahead with big quantum jumps. This article is the first attempt to describe the process of full intuitive creativity as a coherent work of the Quantum Computer of our Subconscious, which works with the waves on our weak nonlinear electromagnetic field (NEMF) (which rules and regulates everything in the body) and the Digital Computer of our Conscious. During the process of full intuitive creativity, the Subconscious and the Conscious work as one whole piece at the same level of over-excitement. The nonequilibrium theory of Prigogine is used to explain this far-from-equilibrium over-excited state and its unpredictable moment of appearing.
\end{abstract}

Keywords: coherent work of subconscious \& conscious
Volume 7 Issue 5 - 2018

Maria Kuman

Holistic Research Institute, Knoxville TN 37923, USA.

Correspondence: Maria Kuman, Holistic Research Institute, Knoxville TN 37923, USA.Email holistecare I @gmail.com

Received: October 01, 2018| Published: October 17, 2018

\section{Full intuitive creativity is over-excitement of both the conscious and the subconscious}

Poincare called the full intuitive creativity a state of "overexcited conscious". ${ }^{1}$ The Russian Shakespeare-the dramas writer Alexander Pushkin- describes his state of creative ecstasy as: "when conscious and subconscious are mixed", when "the conscious cannot succeed to process all that is offered by the subconscious". 2 According to Alexander Pushkin, "creativity is excitement plus inspiration. Inspiration is as necessary in poetry as it is in geometry". ${ }^{2}$ The famous Russian Academic Migdal, who studied creativity, claims that in most cases achieving state of creative ecstasy requires some efforts: "avoiding all the obstacles and rejecting all side thoughts, with consistent and constant efforts, with different side approaches, one have to reach the state of ecstasy, when conscious and subconscious are mixed, when the conscious thinking continue during the sleep and subconscious work is done during the day". ${ }^{3}$ Indeed, for most creative people there is an element of unpredictability when the state of enlightenment will be reached. Also, the creative periods are not everlasting and once they disappear, it is not known when they will come back. However, Alexander Pushkin was not one of them. The writing of Alexander Pushkin seemed to be effortless. He wrought about his political captivity in Borodin: "All days were full with dense and constant full illumination that made the grief and care go away dispersing all the doubts, and one can work 20 out of 24 hours without feeling tired. My Conscious could barely succeed to process all that was offered by my Subconscious, but this created a fabulous feeling of full life and full happiness", ${ }^{2-6}$

\section{Full intuitive creativity requires quantum nonlinear description}

Several scientists attempted to invoke quantum mechanisms in the creative work of the mind. Such were for example. ${ }^{7-11}$ Goswami's argument for using quantum approach to creativity was: creative ideas always come suddenly and unexpectedly. Is the unpredictability of the moment, in which a creative idea will pop up, enough of an argument for quantum description? ${ }^{12}$ Creativity is unthinkable without excitement and over-excitement, ${ }^{13}$ and the moment when such state of over-excitement will take place is unpredictable. The nonequilibrium theory of Prigogine deals with near-to-equilibrium (excited) states and far-from-equilibrium (over-excited) states. The far-from-equilibrium excited states are neither easy to reach nor leave, and the moment when these states would be reached or left is unpredictable. ${ }^{12}$ It seems that the nonequilibrium theory, which deals with unpredictable events just as the quantum mechanics, could be expected to provide adequate description of the unpredictable process of intuitive creativity. However, according to the nonequilibrium theory of Prigogine, the far-from-equilibrium states could be described only with nonlinear equations, which are more difficult to solve.

Thus, the unpredictability of the moment of full intuitive creativity requires quantum or nonlinear nonequilibrium theory, ${ }^{12}$ or both. ${ }^{14}$ Other authors later used the nonequilibrium theory to describe brain dynamic. ${ }^{15}$

\section{Why quantum and nonlinear approach to intuitive creativity?}

Neurotransmitters are emergency substances pre-synthesized and stored in vesicles at the neurosynaptic junctions of our conscious mind, ready to be released. When a necessity arises, the neurotransmitters at all neurosynaptic junctions are released simultaneously like a puff. "The creative people are more easily excitable, something that could be based on abundance of neurotransmitters". ${ }^{19}$ "Glutamates are believed to be the neurotransmitters responsible for excitation". The release of neurotransmitters in the Conscious Mind in puffs allows fast and efficient energy pumping of the Conscious in quants. This allows the Conscious to speedily reach the level of over-excitation of the Quantum Computer of the Subconscious Mind, which operates with the waves of the human Nonlinear Electromagnetic Field (NEMF), and rules and regulates all the organs and everything 
else in the body. ${ }^{20}$ Since the emotional brain called Limbic System is in the Subconscious, the emotional excitement must start in the Subconscious. Obviously the same emotions, which activate the Quantum Computer of the Subconscious, trigger the quantum release of the neurotransmitters glutamates in the Conscious and this quantum release allows the united work of Conscious and Subconscious at the same level of over-excitement, which is the basis of full intuitive creativity. ${ }^{16,17}$

According to the nonequilibrium theory of Prigogine, entrainment is a frequency (energy) resonance between two states, which is specific only for nonlinearly related units. Since it seems that the emotionally over-excited Subconscious entrains the over-excited Conscious to work at the same level of over-excitement, ${ }^{18}$ full intuitive creativity would require nonlinear description. As a whole, intuitive creativity would require both quantum and nonlinear description. If so, the parameter of interaction of the Conscious $\mathrm{C}$ and the Subconscious $\mathrm{S}$, can be expected to depend nonlinearly on the concentration of the released neurotransmitters of excitation glutamates in the Conscious.

\section{Emotional individuals are more intuitively creative}

Thus, achieving a state of full intuitive creativity depends on the emotional excitability of the individual. It was found that individuals, who are easily excitable, emotionally sensitive, or highly emotional, have large amounts of glutamates released at each neurosynaptic junction. ${ }^{19}$ It was also found that highly emotional individuals have better memory because the abundant neurotransmitters of excitation, glutamates, enhance memorizing. ${ }^{21}$ Since long-term conscious memory requires formation of new neuronal connections, the more information memorized, the larger the number of neurons and neurosynaptic junctions in the brain. If so, highly emotional individuals will not only have larger amounts of glutamates released at each neurosynaptic junction, they will also have a denser set of neurons and neurosynaptic junctions because of their larger memory storage. As a result, the conscious brain of emotional individuals would easily achieve a higher level of excitation. Also, clinical research of, ${ }^{22}$ indicated that emotional individuals have better hypnotic abilities, while M.L. Zinn independently reported in 1994 that individuals with high hypnotic ability learn very quickly. ${ }^{22}$ This means that both, hypnotisability and learning ability are related to the abundant release of neurotransmitters of excitation, glutamates, in emotionally sensitive individuals. The author showed in, ${ }^{23}$ that the Quantum Computer of the Subconscious works with holographic images. This means that the full intuitive creativity starts in the Subconscious with exciting emotional images created and stored in the hippocampus and arranged by the amygdala. (Both are in close proximity to the upper large ring of the Limbic System eliciting positive emotions). During creativity all these three subconscious areas will be emotionally excited. ${ }^{24}$ These local excitations will grow to a global excitation of the whole Subconscious. However, there is a barrier with certain height between the Subconscious and the Conscious. For highly emotional individuals, who have larger amount of glutamates released in the Conscious per excitation, the amount of energy $\Delta$ Ei' added at each excitation $i$, will be larger. As a result, the emotional individuals will overcome the barrier between the Conscious and the Subconscious easier and faster after lesser number of quants of excitation. Therefore, the abundant release of glutamates in emotional individuals will not only make them learn quickly, they will be extremely intuitively creative and will easily achieve the state of creative ecstasy. The abundant release in large puffs of neurotransmitters of excitation, glutamates, will energetically pump their Conscious with quants of energy allowing it to reach the level of emotional excitement of the Subconscious. This will allow the Subconscious and the Conscious to work as one whole piece at the same level of over-excitement.

\section{Quantum nonlinear approach to full intuitive creativity}

In a state of full intuitive creativity, the Conscious $\mathrm{C}$ and the Subconscious S work at a level of over-excitement, which is higher than the top of the barrier B between them. As said, the emotional Subconscious, which house the emotional brain called Limbic system, gets excited first and entrains the Conscious to work at the same level of over-excitement. Since entrainment is specific only for nonlinearly related oscillators, ${ }^{16}$ full intuitive creativity would require nonlinear description. However, the Conscious and the Subconscious are not simple oscillators. The Conscious brain has a few major representing frequencies called alpha, beta, theta, and delta waves. But entrainment of two conscious brains was experimentally observed as synchronized sleeping patterns of wife and husband, ${ }^{17}$ and mother and child. ${ }^{18}$ Therefore, two Conscious brains could be entrained, even when each of them is a complex system of nonlinearly related oscillators. Many creative people describe their state of full intuitive creativity as a performance at the top of their abilities, when one can accomplish more for less time. This means that everything in a state of full intuitive creativity is done much faster. Since from hypnosis it is known that when the Conscious is put to sleep, the rate of computation of the Subconscious is much faster, the faster operation in a state of full intuitive creativity is additional proof that the Subconscious is the one that has entrained the Conscious to work at the same level of over- excitement with the same high speed. ${ }^{25-27}$

This means that in the Subconscious is the main Quantum Computer operating with the waves of our nonlinear electromagnetic field (NEMF) with much higher speed, while the Conscious is our Digital Computer operating with lower speed on the biochemical principle. ${ }^{14}$ The excitation starts in the Quantum Computer of the Subconscious and when it is high enough it entrains the excited Conscious, which is our operative memory. This allows a state of ecstasy to be reached, which feels like enlightening. However, the moment when this state of full intuitive creativity would be reached is unpredictable. Element of surprise is specific for all events related to the Subconscious, be it intuitive enlightenment (or spontaneous remission). The intuitively creative persons could never explain how they have done it. If the discovery is a mathematical theory or formula and it came as enlightenment from the Subconscious, usually it takes a lot of time to prove it following the laws of logic of the Conscious. The intuitively created inventions or theories are leaps into the future from the viewpoint of the present level of knowledge because the Subconscious does not obey the causality principle and allows quantum jumps to the future (and the past) ${ }^{23}$ If these intuitive theories had to be derived based on present knowledge and laws of logic, they would perhaps come 50 to $100 y$ years later, and usually it takes 50 to 100 years for them to be really appreciated.

\section{Biophysical and biochemical measures of creative abilities}

Hypnosis is putting the Conscious to sleep to access the Subconscious. Hypnotizability was first measured electrometrically 
in $1950,{ }^{24}$ and it measures how easy it is to reach the Subconscious. Since full intuitive creativity starts with excitation and overexcitation of the Subconscious, the electrochemical measurements of hypnotizability (which determines how easy it is to reach the Subconscious) can also be used as a biophysical measure of full intuitive creativity. Now (in 2018), when we know that the Quantum Computer in the Subconscious works with the waves of the human nonlinear electromagnetic field (NEMF), ${ }^{23}$ it is understandable why the access to the Subconscious could be measured electrometrically. However, in 1950 the electrometrical measurement of hypnotizability (determining the access to the Subconcious) was intuitive insight, which was a jump into the future. Biochemical measure of full intuitive creativity would be measurement of the total amount of secreted glutamates or their final metabolite $\mathrm{N}_{2} \mathrm{O}$ (laughing gas). If $\mathrm{N}_{2} \mathrm{O}$ is marked radioactively, full intuitive creativity can be measured with PET scanners. Such measurements would be safe because the half lifetime of the radioactive isotopes of $\mathrm{N}$ and $\mathrm{O}$ is 2 and $10 \mathrm{~min}$. correspondingly, which means that the radioactive isotopes would be halfway excreted in $10 \mathrm{~min}$. The more emotional is an individual, the higher is the amount of depleted neurotransmitters of excitation, glutamates, and the higher is his intuitive creativity. Since the neurotransmitters are electrically charged, electric measurement could determine not only the emotional sensitivity of the individual, but also his creative potential, or his chances for achieving a state of ecstasy or full intuitive creativity. ${ }^{28,29}$

\section{Conclusion}

The fact that the measured volt-ampere characteristics of the brain are of hysteresis type,$^{14}$ means that they are nonlinear and would require a nonlinear description. On the other hand, the neurotransmitters are released in the brain in puffs or quanta, which means necessity of quantum description. As a whole the mind and the brain would require quantum nonlinear description. In this article, the process of full intuitive creativity was described as a state, in which the Conscious and the Subconscious work as one piece at the same level of global over-excitement.

\section{Acknowledgements}

None.

\section{Conflict of interest}

The authors declared that there is no conflict of Interest.

\section{References}

1. Poincare H. Science and Hypothesis. Dover Publisher; 1952.

2. Meilakh BS. Talisman: Kniga o Pushkine. Sovremennik; 1975.

3. At the Border of Science and Art, Leningrad; 1971.

4. Talant Pisatelia: Processy Tvorchestva, Sov. Pisatel, Leningrad; 1969.
5. Pushkin VS. Evristika-Nauka o Tvorcheskom Mishlenii. Moscow; 1967.

6. Rosner S Abt LE. The Creative Expression, North River Press; 1976.

7. Kant I. Essays. Europe; 1952.

8. Uchtomski in: Ponomar'ev AJ. Psychika i Intuitsia (Russ.). Moscow; 1967.

9. Kak S. Quantum Neuronal Computing, in: Advances in Imaging and Electron Physics. In: Hawkes P, editor. Acad Press;1995;64:259-313.

10. Goswami A. The Journal of Creative Behavior, 1989;22:9-30.

11. Nobili R. Effect of dissipation on quantum coherence. Phys Rev. 1985;A32:3618-3626.

12. Wolf FA. Starwave. NYC: MacMillan; 1984

13. Stuart CIJM, Takahashi Y, Umezawa M. Mixed-system brain dynamics: Neural memory as a macroscopic ordered state. Foundation of Physics.1979;9(4):301-329.

14. Prigogine I. Order out of Chaos. Bantam Books, Toronto, New York, London, UK; 1984.

15. From Being to Becoming. Freeman and Co; 1980.

16. Piechovski, Cunningham. Journal of Creative Behavior. 1985;19:153.

17. Kuman M. A Guide to the Mind's Secrets. Health \& Happiness Books; 1993.

18. Freeman W, Vitiello G. Nonlinear Brain Dynamic as Manifestation of Underlying Many Body Dynamics. Physics of Life Reviews. 2006;3:93-118.

19. Minorski N. Nonlinear Oscillations. Prinston NJ: Van Nostrand; 1964.

20. Winfree A. The Timing of the Biological Clocks. Sci American, NY; 1987.

21. Luce GG. Biological Rhythms in Psychiatry and Medicine. NIH; 1970.

22. Restak RM. The Brain, Bantam Books. Toronto, NY, London, UK; 1984.

23. Kuman M. Research in Medicine and Engineering Sciences. 2018;5(2).

24. Squire LR. Influence of prior events on cognitive judgments in amnesia. Psychological Review. 1992;99:195-231.

25. Wickramasekera I. Professional Psychology: Research and Practice. USA. 1986;17:437-447.

26. Clinical Behavioral Medicine: Some Concepts and Procedures. New York: Plenum Press; 1988.

27. Handbook of Clinical Hypnosis. In: Lynn S, Rhuye J, Kirsh I, editors. Washington DC: APA; 1993.

28. Kuman M. International Journal of Complementary and Alternative Medicine. IJCAM. 2018;11(4)

29. Ravitz L. Elecrometric Correlates of Hypnotic State. Science. $1950 ; 112: 341$. 